This item was submitted to Loughborough's Research Repository by the author.

Items in Figshare are protected by copyright, with all rights reserved, unless otherwise indicated.

\title{
Modelling thin film growth in the Ag-Ti system
}

PLEASE CITE THE PUBLISHED VERSION

https://doi.org/10.1016/j.susc.2018.08.020

PUBLISHER

(C) Elsevier

VERSION

AM (Accepted Manuscript)

PUBLISHER STATEMENT

This paper was accepted for publication in the journal Surface Science and the definitive published version is available at https://doi.org/10.1016/j.susc.2018.08.020.

\section{LICENCE}

CC BY-NC-ND 4.0

\section{REPOSITORY RECORD}

Zhou, Ying, Adam L. Lloyd, Roger Smith, and Steven D. Kenny. 2019. "Modelling Thin Film Growth in the Ag-ti System". figshare. https://hdl.handle.net/2134/35030. 


\title{
Modelling thin film growth in the Ag-Ti system
}

\author{
Ying Zhou ${ }^{\mathrm{a}, *}$, Adam L. Lloyd ${ }^{\mathrm{a}}$, Roger Smith ${ }^{\mathrm{a}}$, Steven D. Kenny ${ }^{\mathrm{b}}$ \\ ${ }^{a}$ Department of Mathematical Sciences, Loughborough University, Leicestershire, LE11 \\ 3TU, United Kingdom \\ ${ }^{b}$ Department of Materials, Loughborough University, Leicestershire, LE11 3TU, United \\ Kingdom
}

\begin{abstract}
Simulations of thin film growth in the Ag-Ti system are presented using molecular dynamics combined with an adaptive kinetic Monte Carlo method (AKMC) with a modified embedded atom potential fit to ab initio data for the surface energies. For the model, atoms are assumed to deposit normally with a kinetic energy of $1-3 \mathrm{eV}$, with a typical deposition rate of around 10 monolayers per second, similar to what might be expected in a sputter deposition process. For the growth of $\mathrm{Ti}$ on the $\mathrm{Ag}$ (100) and $\mathrm{Ag}$ (111) surfaces, the Ti adatoms prefer to exchange with the original surface layer atoms creating a mixed $\mathrm{Ag} / \mathrm{Ti}$ surface. On a silver substrate, up to four mixed layers need to be formed before a pure Ti layer is obtained. Conversely, simulations of Ag depositing onto $\mathrm{Ti}$ (0001) showed that in the initial phase of growth, the Ag adatoms prefer to be separated before a complete first layer of $\mathrm{Ag}$ was obtained in a close-packed structure. The implementation of a super-basin method within AKMC allowed the simulation of $0.4 \mathrm{~s}$ of $\mathrm{Ti}$ growth on the Ag substrates, with up to 3 new layers added.
\end{abstract}

Keywords: titanium, silver, thin film growth, long-time scale dynamics, super basin method

\section{Introduction}

Multilayer thin films containing silver are used in the glass industry to produce low emissivity (low-E) coatings. The silver, a few nanometres thick,

\footnotetext{
${ }^{*}$ Corresponding author

Email address: y.zhou3@lboro.ac.uk (Ying Zhou)
} 
acts as an infra-red blocker and is contained in a multi-layer stack which has many different interfaces. These coatings have good optical properties such as high transmittance and low absorption and are often grown commercially using magnetron sputtering [1]. To optimise the coatings requires a knowledge of how the different materials grow. One such interface considered by our group, 2 involved modelling $\mathrm{Ag}$ growth on a $\mathrm{ZnO}$ substrate. This showed that the Ag atoms would cover the $\mathrm{ZnO}$ surface before growing in islands under normal operating conditions. Here the interface between the $\mathrm{Ag}$ and $\mathrm{Ti}$ is considered as $\mathrm{Ti}$ is used to prevent the oxidation of $\mathrm{Ag}$.

Previous work has studied thin films of $\mathrm{Ag}$ on $\mathrm{TiO}_{2}$ or $\mathrm{TiO}$ and these have been the topic of investigation both theoretically and experimentally [3, 4, 5, 6] but Ti-Ag interfaces have been less well studied. One such investigation of the $\mathrm{Ti} / \mathrm{Ag}$ interface was a study [7] where Ag nanoparticles were deposited onto a Ti substrate for potential medical applications but here the growth of $\mathrm{Ti}$ on a silver substrate and $\mathrm{Ag}$ on a titanium substrate are investigated using atomistic modelling techniques. The objective is to understand the growth process on the atomic scale with the ultimate aim of using these materials in optical coatings.

Thin film growth can occur in many different forms. The growth can be layer-by-layer, island or layer plus island [8]. When the deposited metal has a higher surface energy that the substrate metal, the deposited atoms prefer to exchange with the outermost layer atoms. For example when a Ti film is grown on a $\mathrm{Cu}$ substrate, experiments have shown that $\mathrm{Ti}$ can substitute with $\mathrm{Cu}$ surface resulting in a first layer coverage of Ti between $20-30 \%$ [9].

Previous work by our group involving single atom impacts of $\mathrm{Ti}$ onto $\mathrm{Ag}$ surfaces has also suggested that the exchange mechanism can take place [10] whereas it was not observed in Ag impacts on Ti. Other authors have also observed the exchange mechanism e.g. for the Ag-Ni [11] system and the Ti-Al system [12].

To obtain a fuller picture of how the growth occurs, it is necessary to model the effect of successive impacts onto the substrate over experimental time scales that allows for diffusion between successive impacts. This is generally not possible by using molecular dynamics (MD) alone because of the time scales involved and a kinetic Monte Carlo (KMC) approach [13] based on a pre-defined event list can miss important transitions. For example using temperature accelerated dynamics, Sprague et al [14] have shown that different growth patterns can occur when growing $\mathrm{Cu}$ on $\mathrm{Ag}$ if $\mathrm{MD}$ alone is used. In this paper we investigate the initial phases of growth of $\mathrm{Ti}$ on both 
Ag (100) and (111) surfaces and also consider Ag growth on Ti using a hybrid MD and adaptive KMC [15, 16] approach that has been used successfully by our group on other systems such as $\mathrm{Ag}$ growth on $\mathrm{Ag}$ [17] and $\mathrm{Ag}$ on $\mathrm{ZnO}$ $[2]$.

\section{Methodology}

\subsection{Interatomic potentials for the Ti-Ag system}

The growth methodology is expensive computationally and so semi-empirical potentials are used rather than ab initio calculations to describe the system. Originally we investigated two potential models based on the embedded atom approach for bulk Ag-Ti systems [18, 19]. However these existing potentials gave a poor agreement with ab initio calculations performed with SIESTA [20] for surface energies. To overcome this deficiency, a new modified embedded-atom method (MEAM) potential [10] was fitted for Ag-Ti interactions based on the existing 2NN MEAM potential [21, 22] for pure $\mathrm{Ag}$ and pure Ti. Various surface configurations were considered with the result being that the new MEAM potential gave a good agreement with the SIESTA results.

\subsection{Molecular Dynamics}

To model the growth, atoms are assumed to impact normally on the heated substrate at energies of a few eV. Most simulations were carried out at a temperature of $300 \mathrm{~K}$. After each impact the simulation was run for around 10 ps. Typical systems contained up to 1000 atoms arranged in 68 layers with the a free surface and with the bottom two layers held fixed and the next layer coupled to a heat bath at the same temperature of $300 \mathrm{~K}$ using a Berendsen thermostat [23]. The system is arranged with surfaces perpendicular to Cartesian co-ordinate axes with the $y$-axis parallel to the free surface normal. Periodic boundary conditions are applied in the $x$ and $z$ directions. After $10 \mathrm{ps}$ the system is relaxed to its local minimum energy configuration before the adaptive KMC (AKMC) methodology is applied.

\subsection{Adaptive Kinetic Monte Carlo}

The adaptive kinetic Monte Carlo (AKMC) developed at Loughborough uses an open-ended saddle point search algorithm to determine the possible transitions (or another impact event) from the local minimum energy state after the MD step. These searches are conducted on subsystems (termed 
defect volumes) of atoms surrounding defects. Usually the Dimer method 24] was used to determine the pathway out of the basin and to find the approximate location of the saddle point after which the nudged elastic band (NEB) [25] was applied to calculate the transition barriers and obtain the minimum energy path more accurately. The transition rates are calculated via the Arrhenius equation:

$$
r=\tau \exp \left(-\triangle E / k_{B} T\right),
$$

where $\triangle E$ is the activation energy, $k_{B}$ is Boltzmann's constant, $T$ is the temperature and $\tau$ is the prefactor which in our simulation was assumed to be fixed at $10^{13} s^{-1}$ but can in principle be calculated via the Vineyard method [26]. A list of events and their transition probabilities is then produced from these searches. Table 1 indicates that when increasing the number of searches, the elapsed time increases linearly but the number of transitions do not have a significant increase for Ti growth on an Ag (111) surface. However, different systems may require different number of searches. For initial growth of $\mathrm{Ti}$ on $\mathrm{Ag}$ (100) surface and Ag on Ti (0001) surface, a few hundred of searches are enough to find all possible transitions. To balance the efficiency, in our simulations the basic transition search number is 1000 per defect volume and was raised to several thousand when the defect area grew bigger. The found transitions are added to the events list along with the probability of another surface impact event. A deposition rate of typically 10 monolayers per second, corresponds to an equivalent transition barrier of $0.59 \mathrm{eV}$ at the temperature $300 \mathrm{~K}$ for the system sizes studied.

Table 1: Average number of unique transitions found and elapsed time (in seconds) when different number of searches carried out on the two defect clusters shown in Fig. 6 using 24 cores in parallel. There are 54 and 44 atoms in the two defect clusters.

\begin{tabular}{lll}
\hline Number of searches & Transitions found & Time $(\mathrm{s})$ \\
\hline 500 & $4.2( \pm 0.1)$ & $789.2( \pm 4.6)$ \\
1000 & $4.5( \pm 0.2)$ & $1571.8( \pm 7.3)$ \\
2000 & $5.6( \pm 0.3)$ & $3144.6( \pm 9.8)$ \\
\hline
\end{tabular}

Once an event list is obtained, one event will be chosen and the clock advances according to a time [27]

$$
\Delta t=-\frac{\ln u}{\sum_{i} r_{i}}
$$


where $u$ is a random number between 0 and 1 and $r_{i}$ is the rate for each event.

The methodology is under continuous development and has a few additions to that described in some of our previous publications, e.g. [28, 17] and includes a super basin method and a different way of identifying local regions where transition searches can be concentrated. These are termed defect volumes.

\subsection{Defect Volumes}

To save computation time, a subsystem is created when making open ended saddle point searches which surrounds system defects and adatoms; typically a radius of $>4 \mathrm{NN}$ is used. The methodology is described in more detail in [29] but typically defect volumes are created around atoms that differ in co-ordination number from those that would occur in a perfect crystal bulk or surface. If multiple defects exist within a defined distance (double the defect volume radius) from each other, the subsystems are combined to create a larger defect volume.

\subsection{Super Basin Method}

Equation (1) indicates that the transition rate is inversely proportional to the activation energy. The higher the rate is, the more likely the transition would be chosen during the simulation. Hence low-barrier transitions can slow down the simulation leaving the system trapped in a super-basin, an object containing states connected by low barrier transitions from which it would take a long time to escape. The mean rate method [30] is used to calculate an average rate for transitions to occur that escape the super-basin. Super-basins are built on-the-fly by exploring all states connected by lowbarrier transitions and then removing these transitions from the KMC event table allowing only the escaping transitions to be included - hence accelerating the simulation time. The super-basin method implemented in our code is an extension of the on-lattice method described in [2] and the off-lattice method further described in [29] where local basins are built from defect volumes, rather than including the whole system. This allows multiple basins to exist simultaneously. However, local super-basins are treated independently from each other unless separate defect volumes become close enough to be considered a combined volume or a deposition event occurs. In these cases, the existing super-basin transition networks are deleted and are built again 
as the system develops. For the Ag-Ti system the transition barrier threshold for events to belong to all the super-basins was taken as $0.5 \mathrm{eV}$.

\subsection{Substrates}

Three system were considered for the growth simulations. The Ag (100) substrate consists of 8 layers with 128 atoms per layer. The Ag (111) substrate has 6 layers with 132 atoms in each layer. There are 10 layers and 100 atoms in each layer for the $\mathrm{Ti}$ (0001) substrate.

\section{Results}

\section{1. $\mathrm{Ti}$ on $\mathrm{Ag}(100)$}

Transitions and Reaction Pathways

Before carrying out growth simulations we first investigate possible transition associated with small Ti clusters on the Ag surface. If a Ti adatom is placed in the hollow site on the Ag (100) surface, a concerted motion of a Ti atom and an $\mathrm{Ag}$ atom is found by open-ended saddle point searching, with the $\mathrm{Ti}$ being incorporated into the surface and the Ag becoming an adatom. The energy barrier for the exchange is $0.42 \mathrm{eV}$, making this exchange more likely than a further deposition, with a much larger reverse barrier of $1.85 \mathrm{eV}$. In the case of a $\mathrm{Ti}$ dimer on the surface, the energy barrier for one of the atoms in $\mathrm{Ti}$ dimer to replace one $\mathrm{Ag}$ atom is $0.30 \mathrm{eV}$. This reduces even further for a Ti trimer with a barrier of $0.09 \mathrm{eV}$. The barrier for a Ti atom to hop on the surface from one hollow site to the adjacent hollow site via a bridge site is $0.71 \mathrm{eV}$. Hence it is more favourable for the deposited Ti atoms to exchange with the outermost layer $\mathrm{Ag}$ atoms.

After this exchange process, the Ag adatom can rotate around the Ti with a barrier of $0.47 \mathrm{eV}$ (Fig. 17) but to hop away to another hollow site requires $0.81 \mathrm{eV}$ (Fig. 1 b), larger than on the pristine surface. The relative rates for the various processes are given in Table 2. The rate for the Ti exchange (8.3 $\left.\times 10^{5} s^{-1}\right)$ is far higher than the deposition rate $\left(1280 \mathrm{~s}^{-1}\right)$ and the surface hop diffusion rates and indeed when carrying out growth simulations it was found that in the initial stages of deposition, the majority of deposited $\mathrm{Ti}$ adatoms exchanged with the Ag surface atoms. Since there is a factor of $>$ 100 difference in the rates shown in Table 2, the system will be dominated by the rotating of the Ag atom about the subsurface Ti and the KMC clock will advance slowly. This suggests that the super-basin methodology could be 
used in growth simulations to speed up the simulation when these rotations occur.
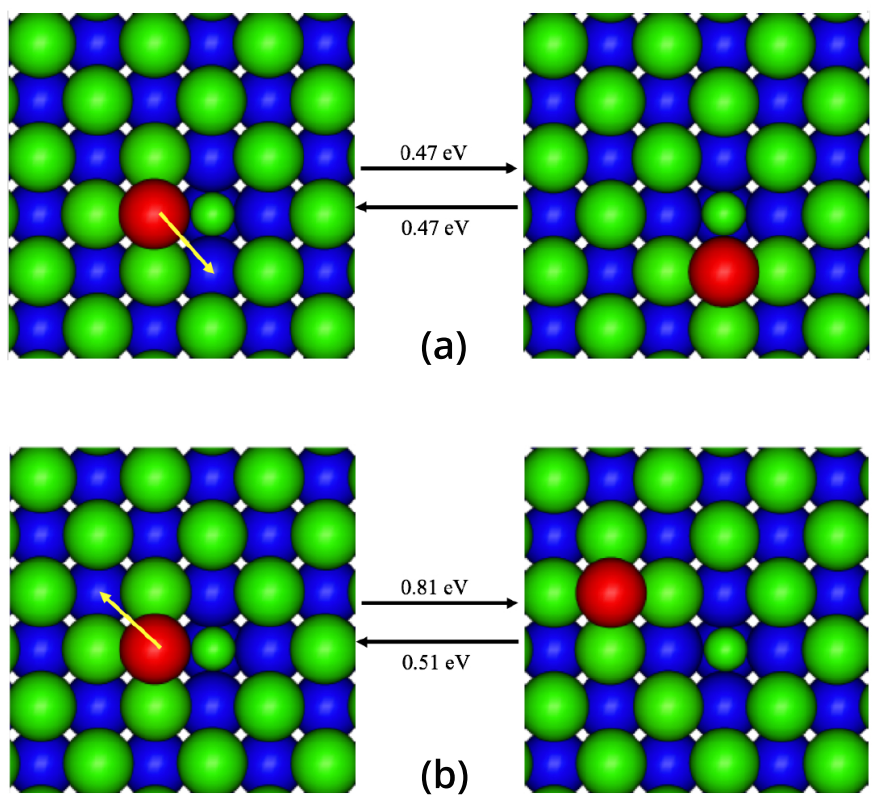

Figure 1: Energy barriers and reverse barriers for single Ag atom to hop on the Ag (100) surface with one Ti in the surface layer. Atoms are coloured by height. The big spheres are Ag and small spheres are Ti. Yellow arrows indicate the direction of transitions..

Table 2: Rates for deposition event and transitions for Ti exchange, rotation around a $\mathrm{Ti}$ atom and hop away from a Ti atom at $300 \mathrm{~K}$.

\begin{tabular}{lll}
\hline Event & $E_{B}(\mathrm{eV})$ & Rate $\left(s^{-1}\right)$ \\
\hline Deposition & 0.59 & $1.28 \times 10^{3}$ \\
Exchange & 0.42 & $8.3 \times 10^{5}$ \\
Rotation & 0.47 & $1.07 \times 10^{5}$ \\
Hop away & 0.81 & 0.25 \\
\hline
\end{tabular}

When there are two close $\mathrm{Ti}$ atoms in the surface, the barriers for $\mathrm{Ag}$ adatoms to move around them is less than $0.3 \mathrm{eV}$ and the probability of choosing a deposition event in the KMC routine is even lower. 


\section{Growth Simulations}

The growth simulations were carried out with the super-basin method implemented. As a result the first deposition occurred at KMC step 5 and we were able to simulate up to $230 \mathrm{Ti}$ deposited onto the $\mathrm{Ag}$ (100) substrate. In Fig. 2 we present the final configuration of $\mathrm{Ti}$ on $\mathrm{Ag}$ (100) by AKMC after 172 milliseconds of real time at a deposition energy of $1 \mathrm{eV}$. The simulation took about one week to finish with 48 cores running in parallel. Fig. 2 shows the surface layer of the substrate is nearly complete with only two vacancies with the first new layer having three vacancies. The percentage of $\mathrm{Ti}$ in the original surface layer is $60 \%$ and in the first new layer the percentage is $49 \%$. The third is about to form and we expect that with more Ti atoms deposited, the Ag atoms will continue to exchange with the deposited $\mathrm{Ti}$.

Another simulation in which the deposition energy is $3 \mathrm{eV}$, is shown in Fig. 3. This simulation was run for 157 milliseconds with $169 \mathrm{Ti}$ atoms deposited. The slight increase in energy results in many atoms depositing on off-lattice sites so that the saddle point search regions are then very large. Hence further application of the off-lattice AKMC requires a huge number of computationally expensive transition searches to find enough transitions belonging to the defect volume for the results to be statistically meaningful.

In order to predict how many mixed layers would be formed before a pure Ti layer is found, would require considerably more computational power and as larger 'defect regions' form the simulation also slows. Thus in order to approximately determine the extent of the mixed region, we have conducted MD only at enhanced deposition rates, ignoring transitions between impacts. MD was run for 5 to 20 pico seconds and relaxed before the next deposition. A total of 400 atoms were added to the system. Although this methodology misses some of the atom exchanges it still shows that over four layers have to be formed, for impact energies less than $20 \mathrm{eV}$, before we get a complete new layer of $\mathrm{Ti}$.

\section{2. $\mathrm{Ti}$ on $\mathrm{Ag}(111)$}

\section{Transitions and Reaction Pathways}

The exchange mechanism for a single Ti adatom to join the Ag surface layer involves three atoms (one $\mathrm{Ti}$ and two Ag atoms) while only two atoms are involved on the $\mathrm{Ag}$ (100) surface. Fig. 4 a shows that when the Ti atom joins the outermost layer, it takes the place of an $\mathrm{Ag}$ surface atom while this $\mathrm{Ag}$ atom pushes an adjacent $\mathrm{Ag}$ atom up onto the surface. The energy barrier is $0.45 \mathrm{eV}$ for this exchange mechanism. 


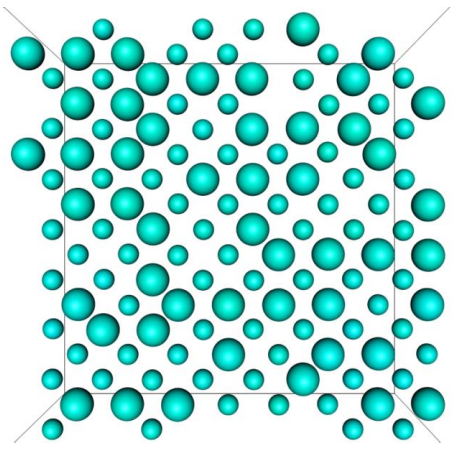

(a) surface layer

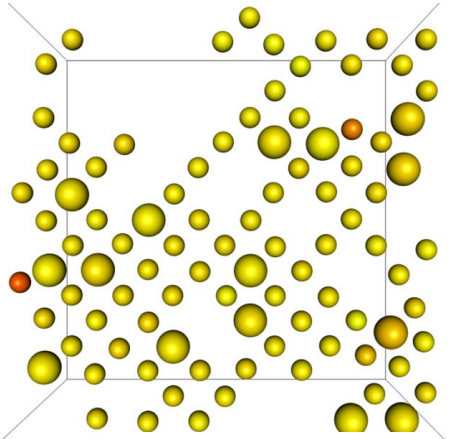

(c) second layer

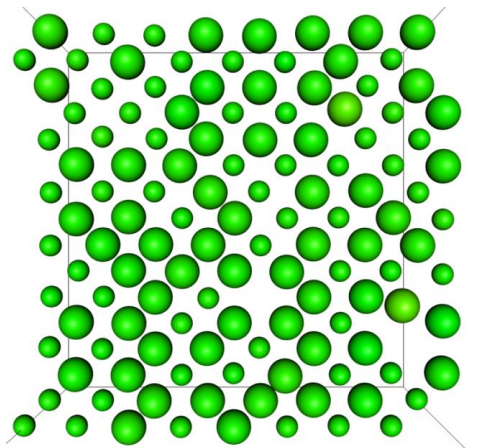

(b) first layer

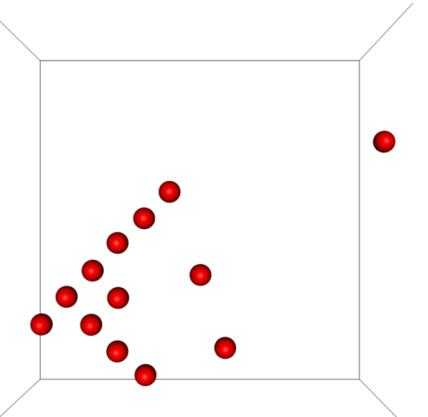

(d) third layer

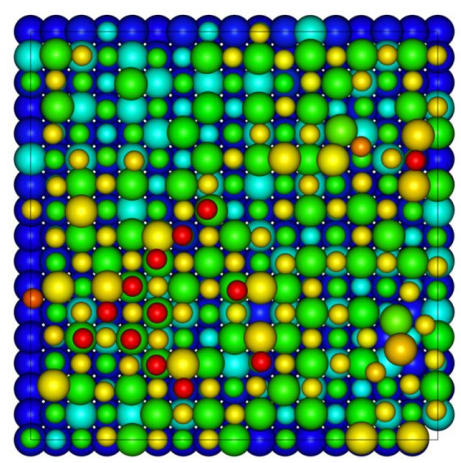

(e) top view

Figure 2: The positions of $\mathrm{Ti}$ atoms deposited onto the $\mathrm{Ag}$ (100) surface at $1 \mathrm{eV}$ using $\mathrm{MD}+\mathrm{AKMC}$. Atoms are coloured by height. The big spheres are Ag and small spheres are Ti. Four mixed layers are shown separately from left to the right and top to the bottom. 


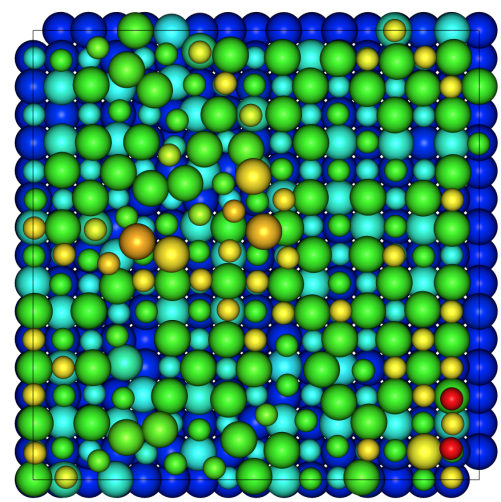

Figure 3: The positions of Ti atoms deposited onto the Ag (100) surface at $3 \mathrm{eV}$ using MD + AKMC. Atoms are coloured by height. The big spheres are Ag and small spheres are Ti. Four mixed layers are shown separately from left to the right and top to the bottom.

When a Ti-dimer is placed on the surface, one of the Ti atoms exchanges with an $\mathrm{Ag}$ surface atom with a barrier of $0.26 \mathrm{eV}$ (Fig. $4 \mathrm{~b}$ ). The new dimer is then pinned to the $\mathrm{Ti}$ in the surface layer. The barrier is $0.20 \mathrm{eV}$ when an atom from a $\mathrm{Ti}$ trimer joins the outermost layer, displacing an $\mathrm{Ag}$ atom onto the surface (Fig. 4c).

Because growth simulations on this surface showed a tendency for clustering, transition barriers for Ag atoms to jump down from a typical island were also calculated as shown in Fig. 5. The corresponding barriers for $\mathrm{Ti}$ are 0.51 and $1.15 \mathrm{eV}$.

\section{Growth Simulations}

In contrast to the (100) surface $\mathrm{Ti}$ adatoms and dimers are very mobile on the surface with barriers of 0.05 and $0.07 \mathrm{eV}$ respectively. Hence with only one $\mathrm{Ti}$ adatom as the initial configuration, the AKMC simulation will show that the Ti will diffuse across the surface for several hundred KMC steps. Even applying the super-basin method does not speed up the simulation since the whole surface becomes a super-basin. We did a few tests running AKMC simulations by randomly placing 8 to $16 \mathrm{Ti}$ atoms on the the $\mathrm{Ag}$ (111) substrate and running MD for $1 \mathrm{~ns}$ at $300 \mathrm{~K}$. When $12 \mathrm{Ti}$ adatoms were placed randomly on the surface, the atoms rearrange themselves into two separate mixed Ag-Ti clusters. Simulations with different starting configurations showed effectively the same patterns. Thus growth simulations were started from initial surface 

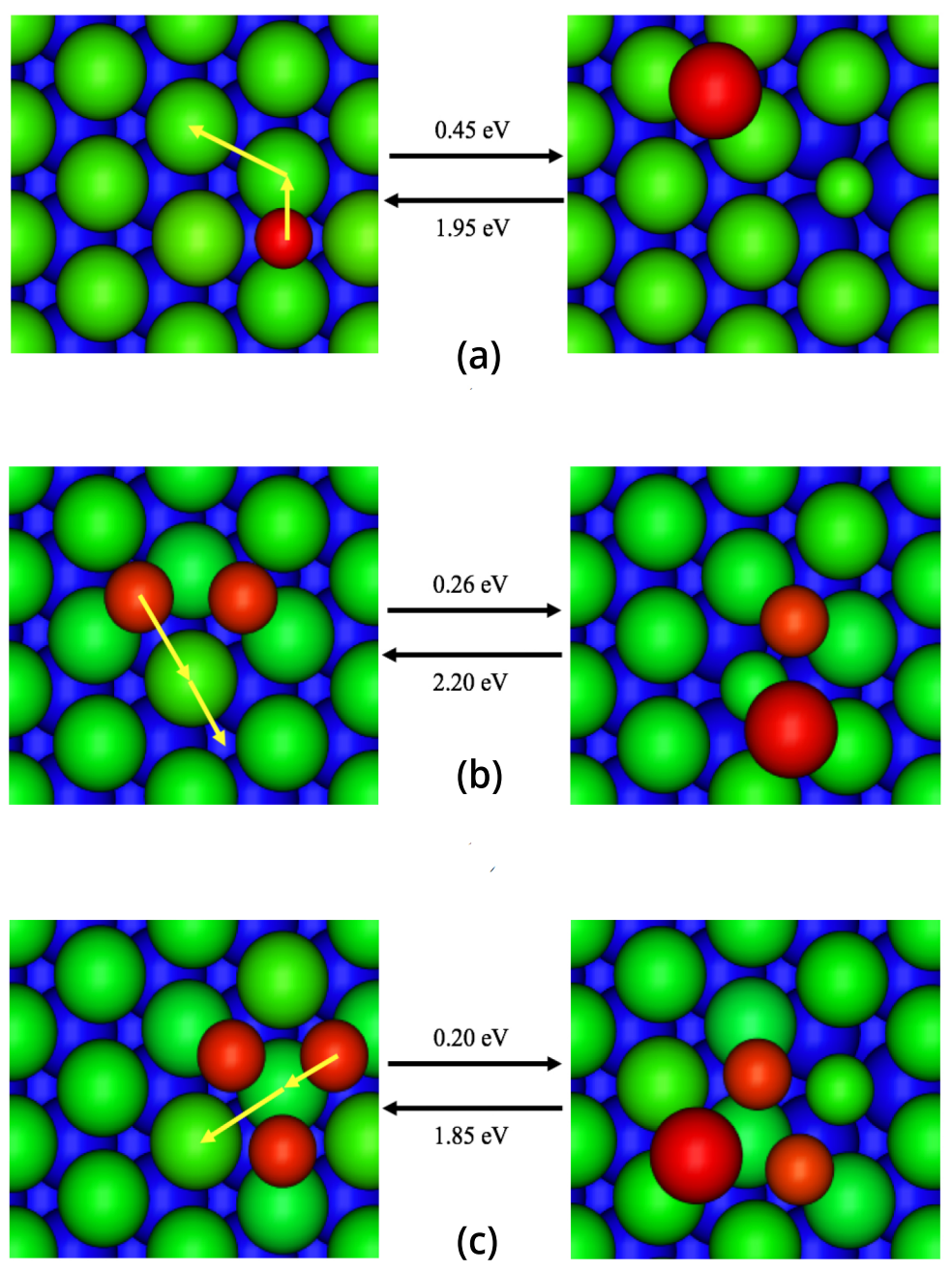

Figure 4: Energy barriers for the exchange of Ti with the surface atom on the Ag (111) surface. Atoms are coloured by height. The big spheres are Ag and small spheres are Ti. Yellow arrows indicate the direction of transitions.

configurations as these. Fig. 6 6 shows clusters (which are then considered as defect volumes) formed during the growth of $\mathrm{Ti}$ on the $\mathrm{Ag}$ (111) substrate after the addition of $17 \mathrm{Ti}$ atoms. The transitions from atoms within these two defect volumes form two local super-basins. During the simulation, each of the local super-basins are explored and the rates for each transition are 

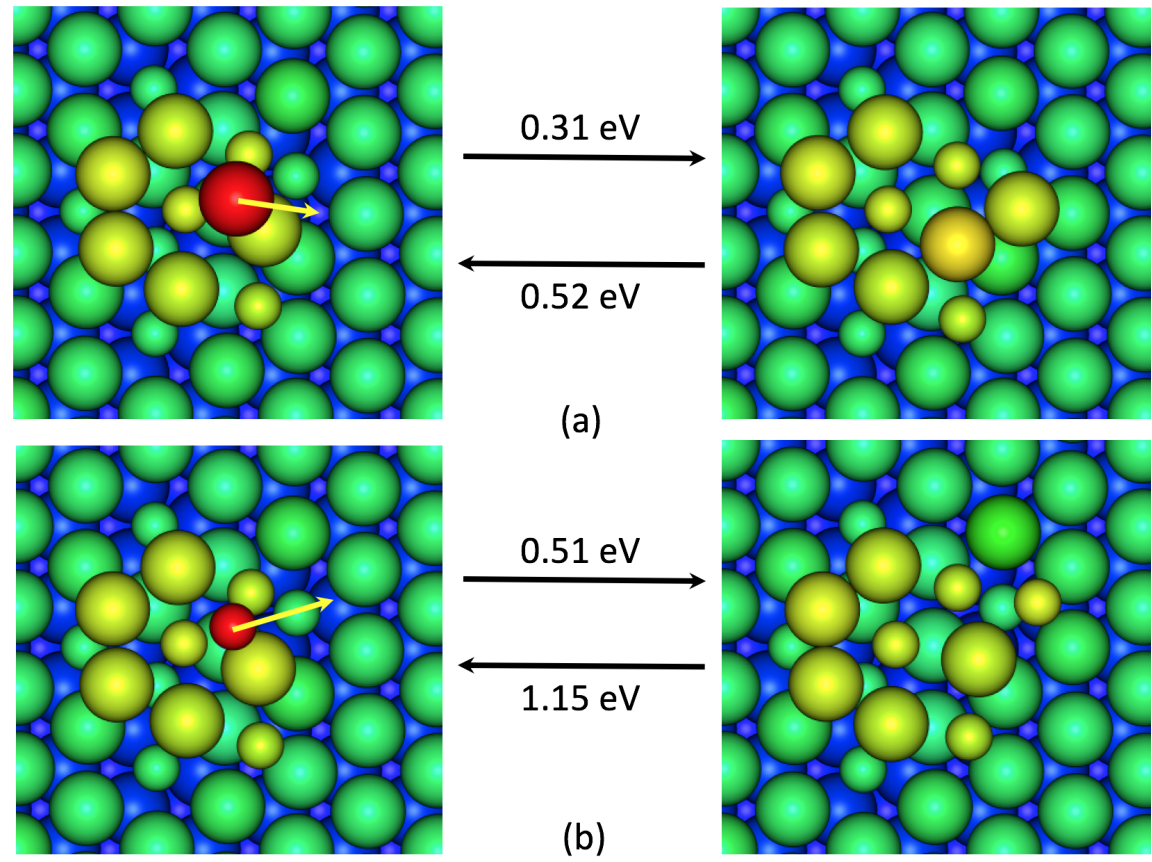

Figure 5: Energy barriers and reverse barriers for Ag and Ti to drop off the step edge of a typical mixed Ti-Ag island on the Ag (111) surface. Large spheres represent Ag and small spheres $\mathrm{Ti}$

modified according to the mean rate method and escaping transition rates are updated and included in the KMC event list.

Fig. 7 shows the system after the addition of $239 \mathrm{Ti}$ atoms. There is one vacancy in the surface layer and the first layer is almost complete with three vacancies. The simulations reached a time of 150 milliseconds. The growth of $\mathrm{Ti}$ on $\mathrm{Ag}$ (111) is first by forming clusters of $\mathrm{Ti}$ and $\mathrm{Ag}$ but by the time the second and third new layer is added, the $\mathrm{Ti}$ adatoms are beginning to form in a close-packed pattern.

\section{3. $\mathrm{Ag}$ on $\mathrm{Ti}(0001)$}

\section{Transitions and Reaction Pathways}

There are two types of hollow sites on the Ti surface: ABC sites and ABA sites shown in Fig. 8 3 . It requires $0.24 \mathrm{eV}$ for an $\mathrm{Ag}$ adatom to move from the ABC hollow to the ABA hollow and the reverse barrier is $0.13 \mathrm{eV}$. These transitions are shown in Fig. 8b. Hence the ABC hollow is preferred over the ABA site. 


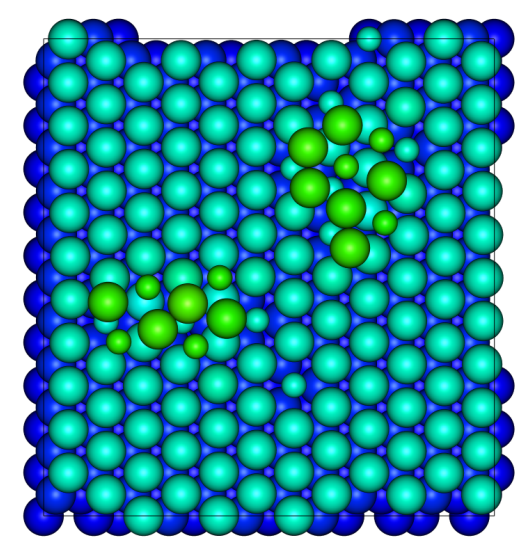

Figure 6: Two local super-basins created after deposition $17 \mathrm{Ti}$ atoms onto $\mathrm{Ag}$ (111) substrate. Atoms are coloured by height. The big spheres are Ag and small spheres are Ti.

An important observation is that when Ag atoms are on a silver island, it is easy for the second layer $\mathrm{Ag}$ atom to drop down the step edge to the Ti surface. Two transitions are shown in Fig. 9 with corresponding energy barriers of $0.03 \mathrm{eV}$ and $0.12 \mathrm{eV}$. Fig. 9 a shows an example of a concerted motion where the second layer $\mathrm{Ag}$ atom pushes one $\mathrm{Ag}$ atom to the $\mathrm{ABA}$ hollow site and then resides on another ABA site.

\section{Growth Simulations}

As with the previous MD + AKMC model, we start the growth with a partially covered surface. $25 \mathrm{Ag}$ atoms were initially placed randomly on the Ti basal plane. The result after the addition of $104 \mathrm{Ag}$ atoms is shown in Fig. 10. The Ag atoms can diffuse individually over the surface but do not cluster and as more $\mathrm{Ag}$ is deposited, a complete layer was formed with a stacking fault. The lattice constants are $2.88 \AA$ and $2.945 \AA$ for $\mathrm{Ag}$ and $\mathrm{Ti}$ respectively indicating that the second layer $\mathrm{Ag}$ atoms do not sit on perfect lattice sites. The energy barrier for diffusion of a single Ag atom on this first added $\mathrm{Ag}$ layer was calculated as only $0.06 \mathrm{eV}$ so the atoms are very mobile. However, as more atoms are added another complete layer begins to form, indicating that islands do not form in the growth of the first 2 layers. After the first two new layers are complete the Ag atoms would be expected to grow in a more island-like form [17] since the further growth is effectively Ag on Ag. 


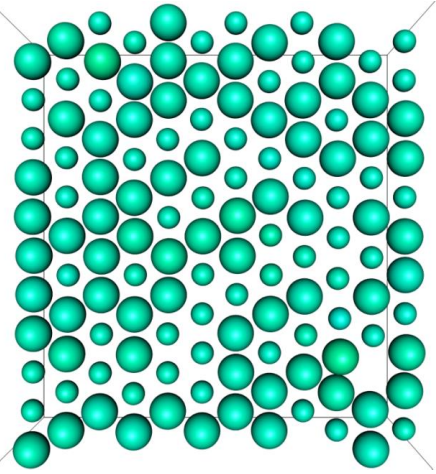

(a) surface layer

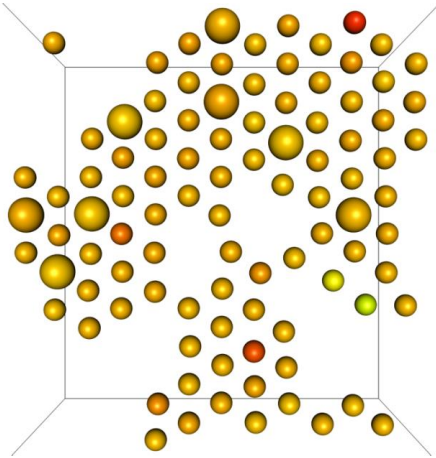

(c) second layer

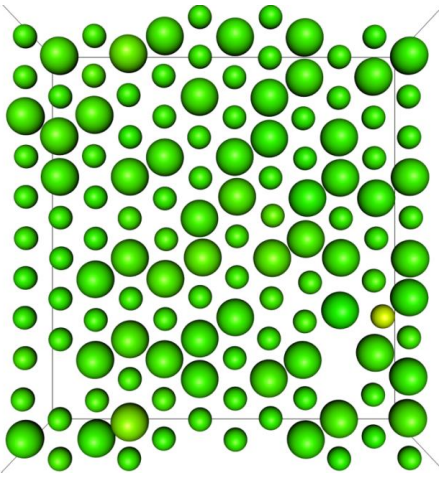

(b) first layer

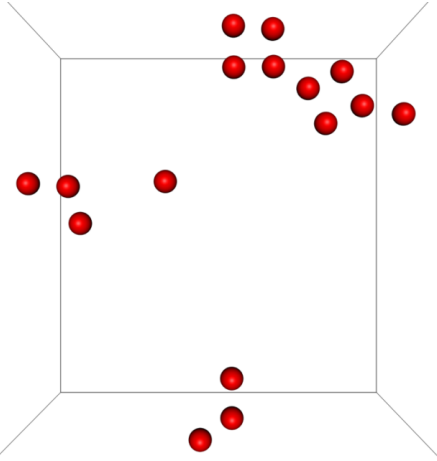

(d) third layer

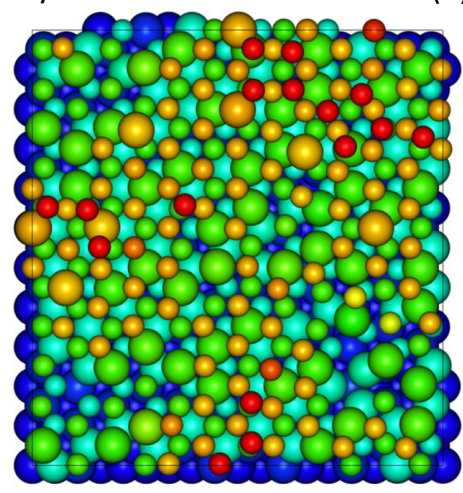

(e) top view

Figure 7: The positions of Ti deposited onto the Ag (111) surface at $3 \mathrm{eV}$ using AKMC + MD. Atoms are coloured by height. The big spheres are Ag and small spheres are Ti. 


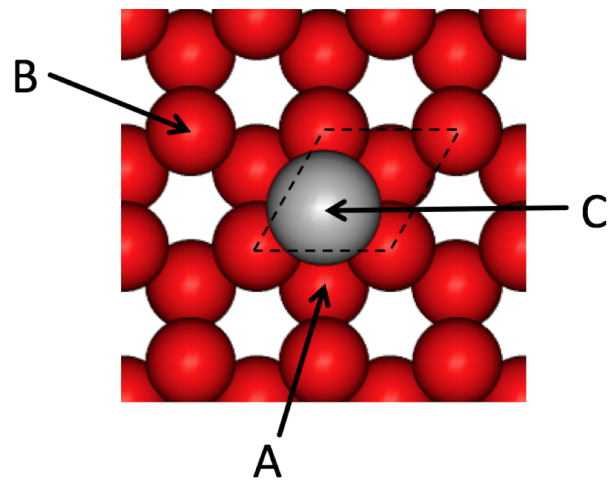

(a)

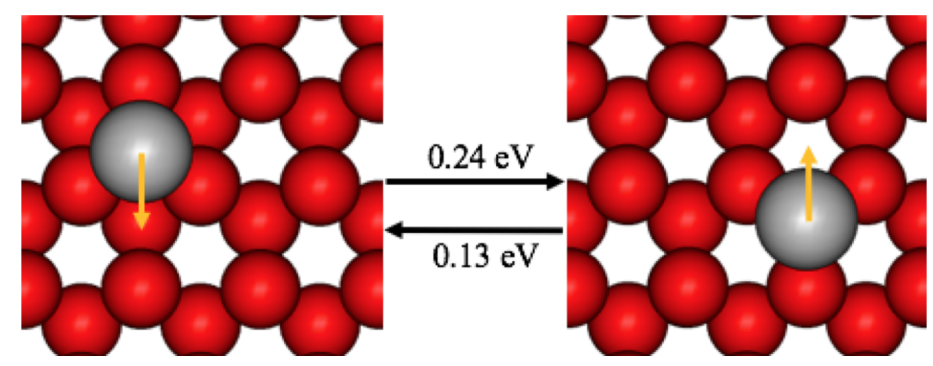

(b)

Figure 8: The diffusion barriers of $\mathrm{Ag}$ on the Ti basal plane. Atoms are coloured by specie. The red spheres are Ti and the grey spheres are Ag. Yellow arrows indicate the direction of the transitions.

\section{Conclusions}

MD combined with AKMC has illustrated the different mechanisms that can occur in the initial stages of growing Ag films on Ti and Ti films on Ag, illustrating the different growth processes that can take place. The methodology is computer intensive but the saddle point searches can be carried out in parallel on different processors so as more computing power becomes available larger and more complex systems can be investigated. Although simulations such as those described here are computationally intensive, they have now reached a stage where they can help scientists involved in producing thin film coatings, because models are increasing in accuracy and deposition conditions can be easily changed on the computer to test new production techniques. 

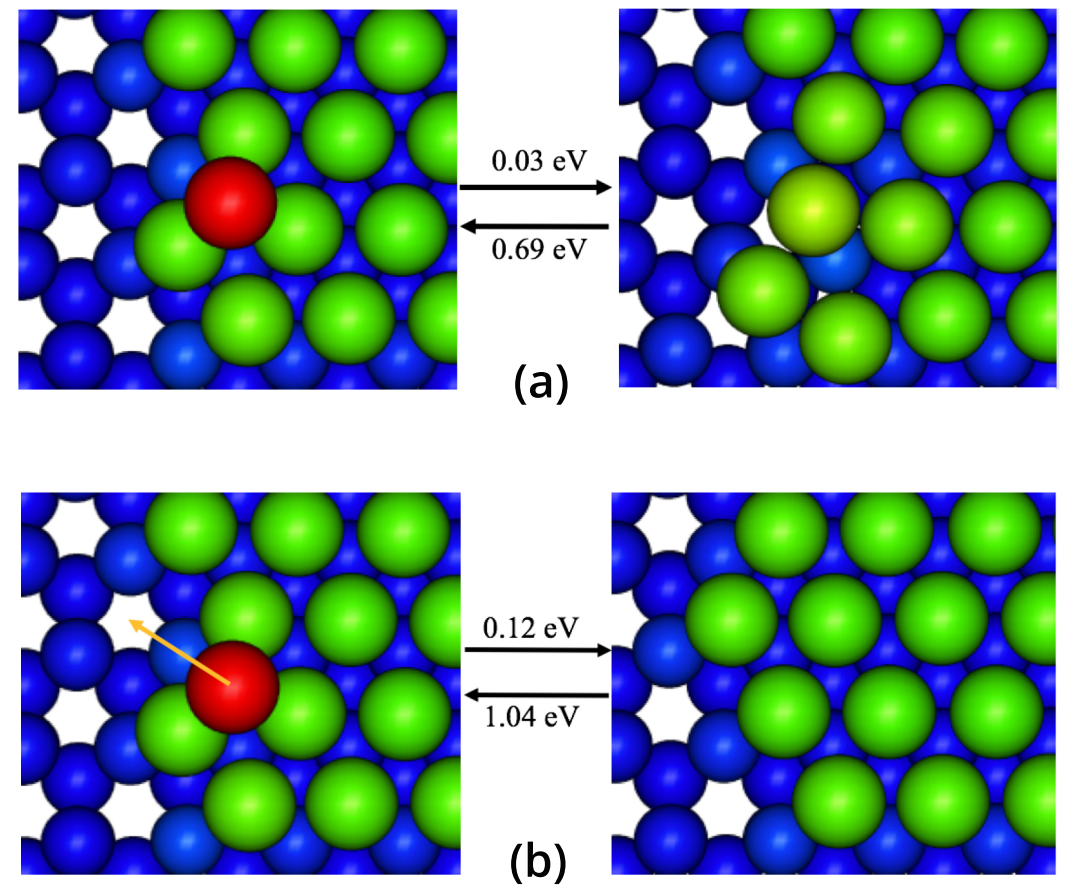

Figure 9: Energy barriers for Ag moving down a step edge from a silver island to a $\mathrm{Ti}$ surface. Atoms are coloured by height. Blue atoms are Ti, green and red atoms are Ag. Yellow arrows indicate the direction of transitions.

\section{Acknowledgments}

This work has been supported by AGC Glass Europe and the Loughborough High Performance Computing unit, funded by EPSRC grant EP/K000055/1.

\section{References}

[1] P. J. Kelly, R. D. Arnell, Magnetron sputtering: a review of recent developments and applications, Vacuum 56 (3) (2000) 159-172.

[2] A. L. Lloyd, R. Smith, S. D. Kenny, Growth of silver on zinc oxide via lattice and off-lattice adaptive kinetic Monte Carlo, Journal of Materials Research 33 (7) (2018) 847-856.

[3] A. Atrei, B. Cortigiani, A. M. Ferrari, Epitaxial growth of $\mathrm{TiO}_{2}$ films with the rutile (110) structure on Ag (100), Journal of Physics: Condensed Matter 24 (44) (2012) 445005. 


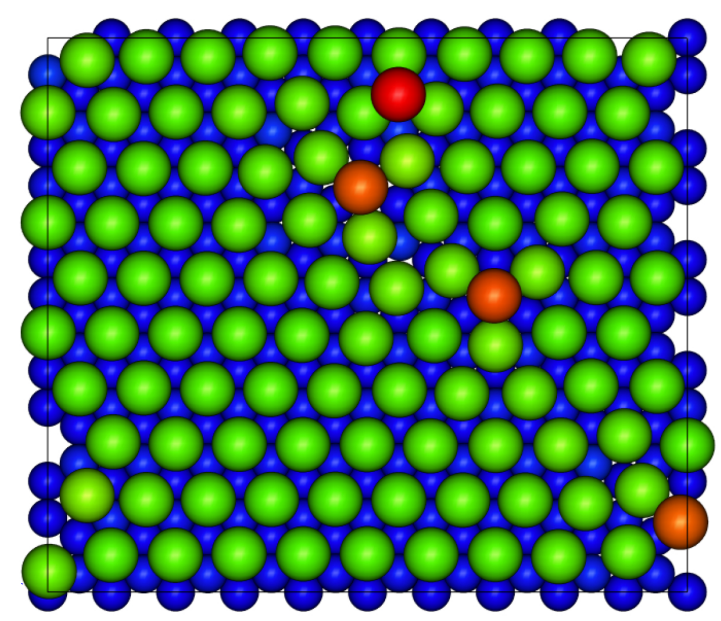

Figure 10: The Ag film structure after the deposition of $104 \mathrm{Ag}$ atoms on $\mathrm{Ti}(0001)$. Atoms are coloured by height. Blue atoms are Ti, green and red atoms are Ag.

[4] A. Romanyuk, P. Oelhafen, Formation and electronic structure of $\mathrm{TiO}_{2}-\mathrm{Ag}$ interface, Solar energy materials and solar cells 91 (12) (2007) 1051-1054.

[5] H. Kaneko, M. Ono, K. Ozawa, K. Edamoto, Growth of ordered titanium oxide films on Ag (100), Solid state communications 142 (1-2) (2007) 32-35.

[6] A. Atrei, A. M. Ferrari, D. Szieberth, B. Cortigiani, G. Rovida, Lepidocrocitelike structure of the $\mathrm{TiO}_{2}$ monolayer grown on $\mathrm{Ag}$ (100), Physical Chemistry Chemical Physics 12 (37) (2010) 11587-11595.

[7] T. Tepper, D. Shechtman, D. Van Heerden, D. Josell, fcc titanium in titanium/silver multilayers, Materials letters 33 (3-4) (1997) 181-184.

[8] J. A. Venables, G. D. T. Spiller, M. Hanbucken, Nucleation and growth of thin films, Reports on Progress in Physics 47 (4) (1984) 399.

[9] M. Caffio, G. Rovida, A. Atrei, Structural investigation of the $\mathrm{c}(\sqrt{2} \times \sqrt{5}) \mathrm{R} 45^{\circ}$ surface alloy formed by $\mathrm{Ti}$ deposition on $\mathrm{Cu}(001)$, Surface science 601 (2) (2007) 528-535.

[10] Y. Zhou, R. Smith, S. D. Kenny, A. L. Lloyd, Development of an empirical interatomic potential for the Ag-Ti system, Nuclear Instruments and Methods in Physics Research Section B: Beam Interactions with Materials and Atoms 393 (2017) 122-125. 
[11] M. Caffio, A. Atrei, U. Bardi, G. Rovida, Growth mechanism and structure of nickel deposited on $\mathrm{Ag}(001)$, Surface science 588 (1-3) (2005) 135-148.

[12] R. R. Zope, Y. Mishin, Interatomic potentials for atomistic simulations of the Ti-Al system, Physical Review B 68 (2) (2003) 024102.

[13] A. Chatterjee, D. G. Vlachos, An overview of spatial microscopic and accelerated kinetic Monte Carlo methods, Journal of computer-aided materials design 14 (2) (2007) 253-308.

[14] J. A. Sprague, F. Montalenti, B. P. Uberuaga, J. D. Kress, A. F. Voter, Simulation of growth of $\mathrm{Cu}$ on $\mathrm{Ag}(001)$ at experimental deposition rates, Physical Review B 66 (20) (2002) 205415.

[15] G. Henkelman, H. Jónsson, Long time scale kinetic monte carlo simulations without lattice approximation and predefined event table, The Journal of Chemical Physics 115 (21) (2001) 9657-9666.

[16] L. Xu, G. Henkelman, Adaptive kinetic monte carlo for first-principles accelerated dynamics, The Journal of chemical physics 129 (11) (2008) 114104.

[17] S. Blackwell, R. Smith, S. D. Kenny, J. M. Walls, Modeling evaporation, ionbeam assist, and magnetron sputtering of thin metal films over realistic time scales, Physical Review B 86 (3) (2012) 035416.

[18] L. Ward, A. Agrawal, K. M. Flores, W. Windl, Rapid production of accurate embedded-atom method potentials for metal alloys, arXiv preprint arXiv:1209.0619.

[19] X. W. Zhou, R. A. Johnson, H. N. G. Wadley, Misfit-energy-increasing dislocations in vapor-deposited CoFe/NiFe multilayers, Physical Review B 69 (14) (2004) 144113.

[20] J. M. Soler, E. Artacho, J. D. Gale, A. García, J. Junquera, P. Ordejón, D. Sánchez-Portal, The SIESTA method for ab initio order-N materials simulation, Journal of Physics: Condensed Matter 14 (11) (2002) 2745.

[21] Y.-M. Kim, B.-J. Lee, M. I. Baskes, Modified embedded-atom method interatomic potentials for Ti and Zr, Physical Review B 74 (1) (2006) 014101.

[22] B.-J. Lee, J.-H. Shim, M. I. Baskes, Semiempirical atomic potentials for the fcc metals $\mathrm{Cu}, \mathrm{Ag}, \mathrm{Au}, \mathrm{Ni}, \mathrm{Pd}, \mathrm{Pt}, \mathrm{Al}$, and $\mathrm{Pb}$ based on first and second nearestneighbor modified embedded atom method, Physical Review B 68 (14) (2003) 144112. 
[23] H. J. Berendsen, J. v. Postma, W. F. van Gunsteren, A. DiNola, J. Haak, Molecular dynamics with coupling to an external bath, The Journal of chemical physics 81 (8) (1984) 3684-3690.

[24] G. Henkelman, H. Jónsson, A dimer method for finding saddle points on high dimensional potential surfaces using only first derivatives, The Journal of chemical physics 111 (15) (1999) 7010-7022.

[25] G. Henkelman, B. P. Uberuaga, H. Jónsson, A climbing image nudged elastic band method for finding saddle points and minimum energy paths, The Journal of chemical physics 113 (22) (2000) 9901-9904.

[26] G. H. Vineyard, Frequency factors and isotope effects in solid state rate processes, Journal of Physics and Chemistry of Solids 3 (1-2) (1957) 121-127.

[27] C. A. M. J. Wert, C. Zener, Interstitial atomic diffusion coefficients, Physical Review 76 (8) (1949) 1169.

[28] C. Scott, S. Blackwell, L. Vernon, S. Kenny, M. Walls, R. Smith, Atomistic surface erosion and thin film growth modelled over realistic time scales, The Journal of chemical physics 135 (17) (2011) 174706.

[29] A. L. Lloyd, Y. Zhou, M. Yu, C. Scott, R. Smith, S. D. Kenny, Reaction pathways in atomistic models of thin film growth, The Journal of chemical physics 147 (15) (2017) 152719.

[30] B. Puchala, M. L. Falk, K. Garikipati, An energy basin finding algorithm for kinetic monte carlo acceleration, The Journal of chemical physics 132 (13) (2010) 134104. 